\title{
Test results of new CR365-PMTs for the Tibet muon-detector array
}

\author{
Ying Zhang ${ }^{* 1}$, J. Huang ${ }^{1}$, D.Chen ${ }^{2}$, L. M. Zhai ${ }^{2}$, Xu Chen ${ }^{1}$, Xiao-Bin Hu ${ }^{1,3}$, Yu-Hui \\ Lin $^{1}$, Hong-Bo Jin ${ }^{2}$, K. Kasahara ${ }^{1,4}$ \\ ${ }^{1}$ Key Laboratory of Particle Astrophysics, Institute of High Energy Physics, Chinese Academy of \\ Sciences, Beijing 100049, China \\ ${ }^{2}$ National Astronomical Observatories, Chinese Academy of Sciences, Beijing 100012, China \\ ${ }^{3}$ Department of Physics, Shandong University, Jinan 250100, China \\ ${ }^{4}$ Research Institute for Science and Engineering, Waseda University, Japan \\ E-mail: vingzhangeihep.ac.cn
}

\begin{abstract}
The Tibet-AS $\gamma$ experiment has a water-Cherenkov muon-detector array (MD) as a part of 3 different arrays with specific purposes. To increase the ability of getting the primary proton, helium and iron spectra up to $10^{16} \mathrm{eV}$ and clarifing their spectral "knee" positions, we need to upgrade current PMT capabiltty in each MD cell. The current dynamic range of the PMTs is 5 to 2000 photoelectrons (PEs) which is enough for the purpose of observation of high-energy celestial gamma rays. However, according to Monte-Carlo simulations, we need a dynamic range of $100-10^{6}$ PEs for the knee problem. For this end, we have a plan of adding two 8-inch-in-diameter PMTs in each MD cell. We have compared the characteristic features between R5912-PMT made by Japan Hamamatsu and CR365-PMT made by Beijing Hamamatsu, and found that CR365-PMT fulfill our requirements. In this paper, test results of the first batch of 20 CR365-PMTs made by Beijing Hamamatsu for the MD array are shown.
\end{abstract}

35th International Cosmic Ray Conference ICRC2017

10-20 July, 2017

Bexco, Busan, Korea

\footnotetext{
* Speaker.
} 


\section{Introduction}

The energy spectrum of cosmic rays is roughly described by a power law over a wide energy range covering more than 10 decades while there exist some structures in the spectrum; this may suggest a remarkable feature of the nonthermal acceleration mechanism of high-energy cosmic rays. Among the power index changes appeared in the all-particle spectrum, the "knee" is the most well known. That is, the power index suddenly steepens from approximately -2.7 to -3.1 at around $4 \times 10^{15} \mathrm{eV}$, resulting in a distinctive "knee" shape in the spectrum[四]. Its origin is an outstanding problem in astroparticle physics[[]][[]]. In order to resolve the origin of the knee, it is critical to measure the primary chemical composition or mass group at energies of $50 \mathrm{TeV}-10^{16} \mathrm{eV}$, especially, to measure the primary energy spectra of individual components and determine a break energy of the spectral index for individual components [[] [四] [回].

In 2014, an important upgrade of the Tibet AS $\gamma$ project was completed, and we then started a new hybrid experiment consisting of an air-shower-core array (YAC-II), a high-density air-shower array (Tibet-III) and a large underground water-cherenkov muon-detector array (MD)[四] [6][ [D]. The MD array now consists of 5 pools set up 2.5 meters underground, each with 16 cells, covering a total area of $\sim 4500 \mathrm{~m}^{2}$. Each cell of the MD array is composed of a concrete water tank with a cubic form of $7.2 \mathrm{~m}$ wide, $7.2 \mathrm{~m}$ long, $1.5 \mathrm{~m}$ deep and two 20-inch PMTs (R3600), lookinng downward, are mounted on the ceiling. The method for obtaining the light-component spectrum of primary cosmic rays at the "knee" energies with the Tibet AS $\gamma$ experiment is described in the paper [ $[\mathrm{G}][\mathbb{[}]$; we found that the MD array plays an important role in mass separation at high energy range according to a Monte Carlo simulation. The current dynamic range of PMTs in each cell of the MD array, designed mainly for high-energy celestial gamma rays detection, is 5 to 2000 photoelectrons (PEs) which is, according to the simulation, too small for the composition measurement in the knee region (a maximum of $10^{6}$ PEs is needed).

Therefore, we decided to add two 8-inch-in-diameter PMTs in each MD cell. We have compared the characteristic features between R5912-PMT made by Japan Hamamatsu and CR365PMT made by Beijing Hamamatsu and found that CR365-PMT fulfill our requirements. Detail comparison is described in the paper[Q].

Since it is the first time for Beijing Hamamatsu to produce this type of 8-inch-in-diameter PMTs largely, we have to test the performance of PMTs seriousely whether they can satisfy our request; we can also provide valuable feedback to the company. In this paper, test results of the first batch of 20 CR365-PMTs made by Beijing Hamamatsu for the Tibet muon-detector array is shown.

\section{The experimental setup}

In order to study the performance of CR365-PMTs, the following quantities have been measured:

1. Single photoelectron spectra(SPE)

2. Dark count rate

3. Ion feedback and after-pulsing

4. Linearity of PMT output signals

5. Non-uniformity 


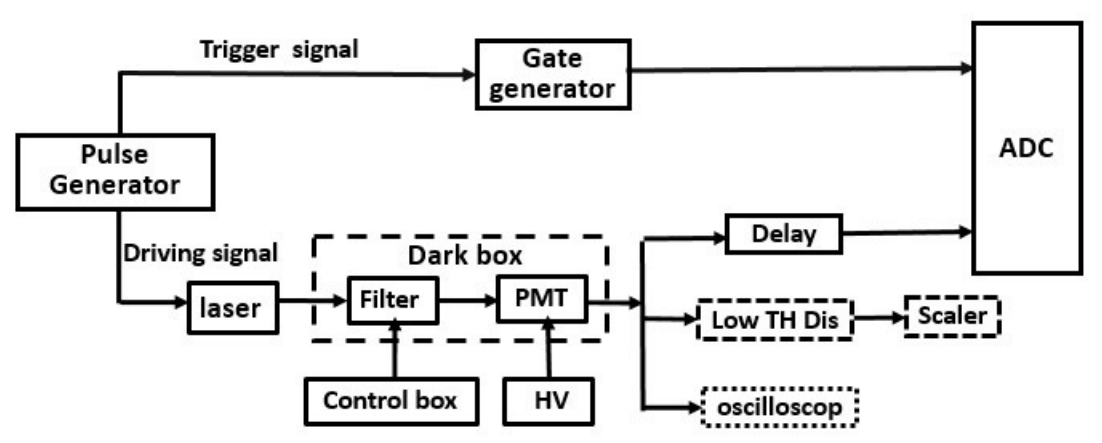

Figure 1: Schematic view of the test system. When we measure 1), 4) and 5), the signals from the PMT are fed into a charge-integrating ADC in a camac crate where they are measured and then read out by the DAQ computer; when we measure 2), the signals from the PMT are fed into a low threshold discriminator and then into a scaler; when we measure 3), the signals from the PMT are then fed into an oscilloscope and then read out by the DAQ computer.

The entire test system is mainly composed of Pulse Generator (Agilent Technologies 81160A), Laser (PiL044SM-SN-513B), Filter, High Voltage power supply (CAEN N1470), Gate Generator (ORTEC GG8020), Low Threshold Discriminator (CAEN N845), Scaler (ORTEC 772 counter), Oscilloscope (Tektronix TDS3032C), ADC (Lecroy 2249A, the resolution is $0.25 \mathrm{pC} /$ count), and data acquisition (DAQ) computer. The test system is schematically shown in Figure 1. The light source used for this test is a pico second laser (Pil044SM-SN-513B) which has very narrow pulse width and high stability. Signal frequency applied to the laser is controlled by the pulse generator (fixed) and the light intensity is controlled by the control box (variable). At the same time, the pulse generator generates the NIM signals to be used as trigger.

Before reaching the PMT, the light passes through the neutral density attenuation filters. With different filters, we are able to change the light intensity. When we measure 1), 4) and 5), the signals from the PMT are fed into a charge-integrating ADC in a camac crate where they are measured and then read out by the DAQ computer; when we measure 2), the signals from the PMT are fed into a low threshold discriminator and then into a scaler; when we measure 3), the signals from the PMT are fed into an oscilloscope and then read out by the DAQ computer.

\section{Test Results}

Using this test system, the single photoelectron (SPE) spectra, dark count rate at $1 / 2$ photoelectron (PE), ion feedback and after-pulsing, linearity of PMT output signals and non-uniformity are measured for the first batch of 20 new CR365-PMTs. Details of each test results are described in the following sections.

\subsection{Single photoelectron spectra}

In order to get the absolute gain of the PMT at a certain voltage, the SPE spectrum is measured. Figure 2(a) shows the SPE spectra of a CR365-PMT corresponding to $10^{7}$ gain. The first peak is the pedestal and the second peak is due to 1 photoelectron events. To quantify the resolution of the 

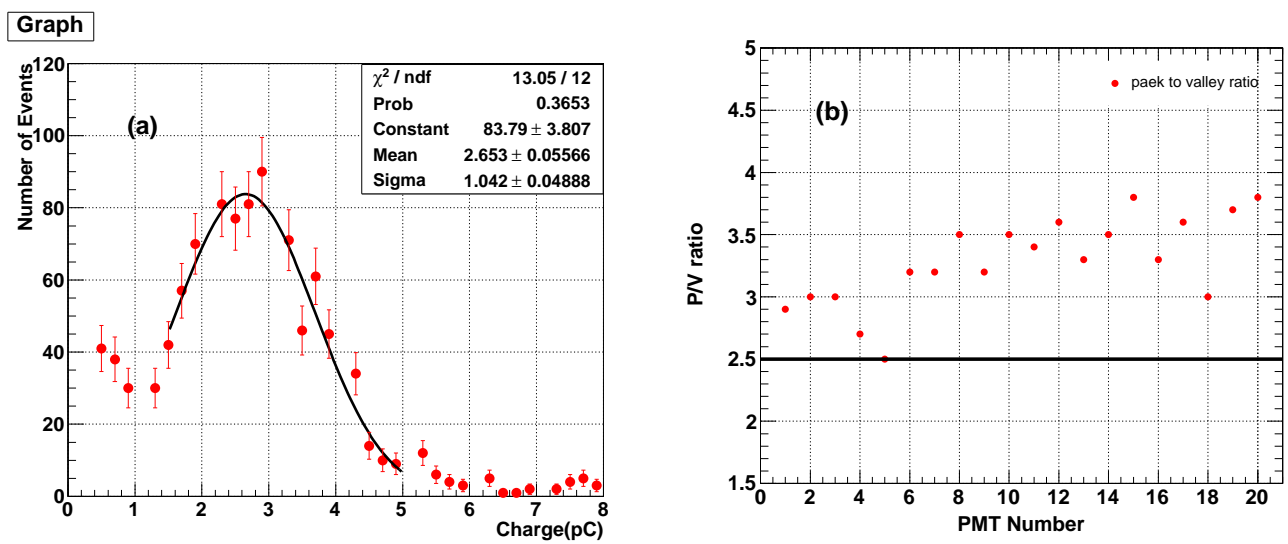

Figure 2: Figure 2(a) shows the SPE spectra of a CR365-PMT corresponding to $10^{7}$ gain. Figure 2(b) shows the measured P/V results of 20 PMTs. The red points indicate the measured P/V value of each PMT and the black line indicate that we require the $\mathrm{P} / \mathrm{V}$ ratio is not less than 2.5 .

single photoelectron spectrum, the peak to valley $(\mathrm{P} / \mathrm{V})$ ratio is used. $\mathrm{P} / \mathrm{V}$ ratio is the ratio of the maximum value of the histogram of the SPE spectrum to the minimum value between the pedestal and the maximum. Figure 2(b) shows the measured P/V results of 20 PMTs. Each red point shows the measured P/V value of each PMT and the black line indicates our minimum requirement of the $\mathrm{P} / \mathrm{V}$ ratio (=2.5). From this figure, we found that the P/V ratio of 20 PMTs meets our requirements.

\subsection{Dark count rate}

The dark count rate is the rate at which signals above a certain threshold are observed in a PMT while no light incident on the photocathode. There is a correlation between the dark pulse rate and the lifetime of a PMT; the smaller the noise of a PMT, the longer its lifetime. To check the dark noise, the data of 20 CR365-PMTs have been measured. The anode dark rate after 2 hours in dark at a gain of $10^{7}$ are both less than $5 \mathrm{k} \mathrm{Hz}$ when the thresholds are set at $1 / 2$ photoelectron, as shown in Figure 3. The performance of dark count rate also meets our requirements.

\subsection{Ion feedback and after-pulsing}

For a newly produced PMT, ion feedback and after-pulsing is a very important parameter, because it is directly related to the cleanliness of the vacuum in a PMT, which is the critical manufacturing problem of all PMTs. Afterpulses could cause a miscalculation of low level signals following a large amplitude pulse and degrade energy resolution. Here, afterpulse percentage is defined as:

$$
A P(\%)=\frac{Q_{a f t e r}}{Q_{\text {signal }}} \times 100 \%
$$

where $Q_{\text {signal }}$ is the charge deposited while the LED is flashing, $Q_{a f t e r}$ is the charge after the main pulse, integrating over $10 \mu \mathrm{s}$. We measured the afterpulse percentage of a CR365-PMT at $10^{7}$ gain and the value is about 4.9\%, as shown in Figure 4(a). Figure 4(b) indicate that the after-pulsing results of 20 CR365-PMTs are less than $15 \%$ which is what we asked for. 


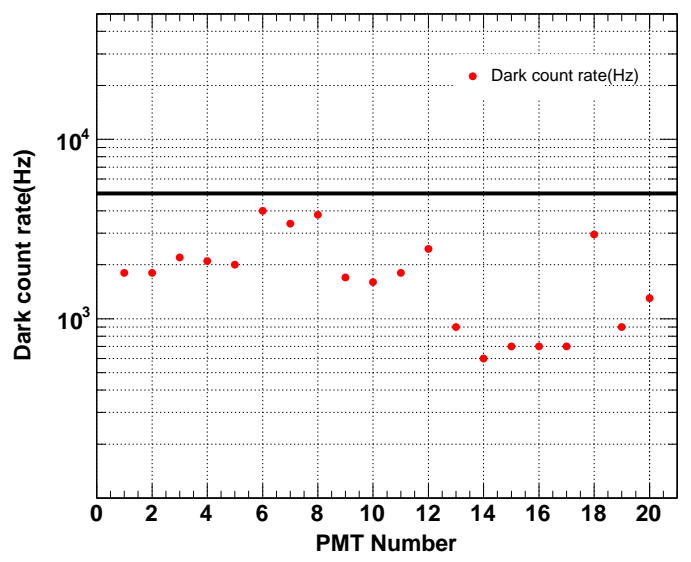

Figure 3: The anode dark rate after 2 hours in dark at a gain of $10^{7}$ are both less than $5 \mathrm{k} \mathrm{Hz}$ when the thresholds are set at 1/2 photoelectron. The performance of dark count rate also meets our requirements.
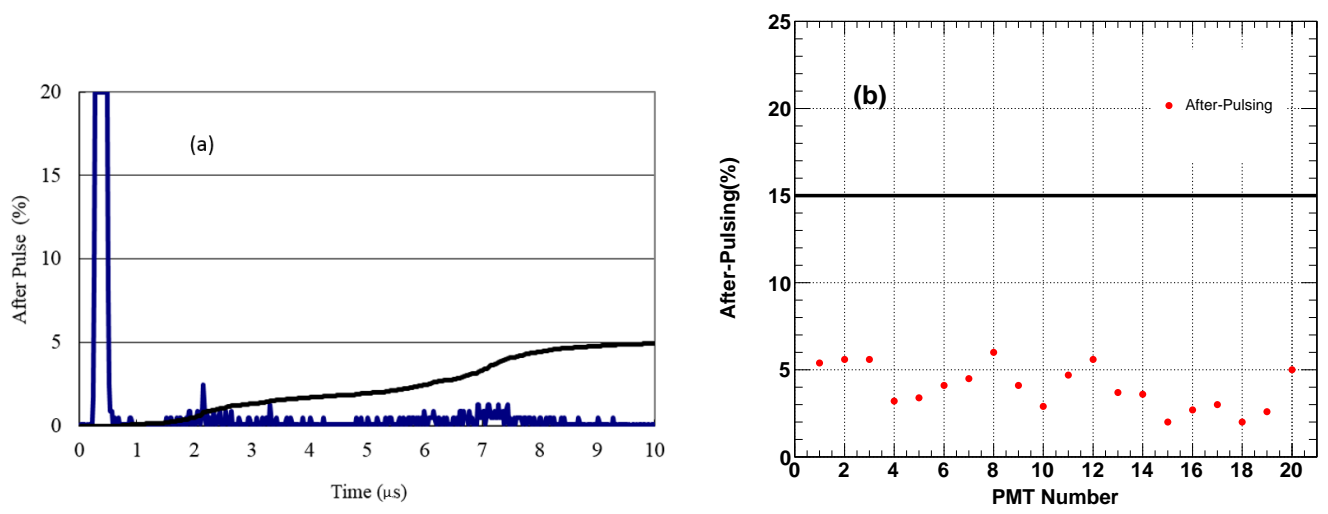

Figure 4: We measured the afterpulse percentage of a CR365-PMT at $10^{7}$ gain and the value is about $4.9 \%$, as shown in figure 4(a). Figure 4(b) cofirmes that the after-pulsing of 20 CR365-PMTs are less than 15\% which is what we asked for.

\subsection{Linearity of PMT output signals}

To quantify the range over which the output response to input light is linear, the linearity of signal output is measured. The "relative light intensity ratio method" is used to measure the linearity of PMT [एU]. In this measurement, an LED operated in a double-pulsed mode is used to provide higher and lower pulse amplitudes alternately. The higher and lower pulse amplitudes are fixed at a ratio of approximately 3:1. If the PMT outputs in response to the higher and lower pulsed light at sufficiently low light levels, the peak current are $Q_{02}$ and $Q_{01}$ respectively, then the ratio of $Q_{02} / Q_{01}$ is proportional to the pulse amplitude, thus

$$
Q_{02} / Q_{01}=3
$$

When we change the filter to increase input light, the subsequent output current increases, the PMT output begins to deviate from linearity. Linearity can be measured by measuring the ratio 

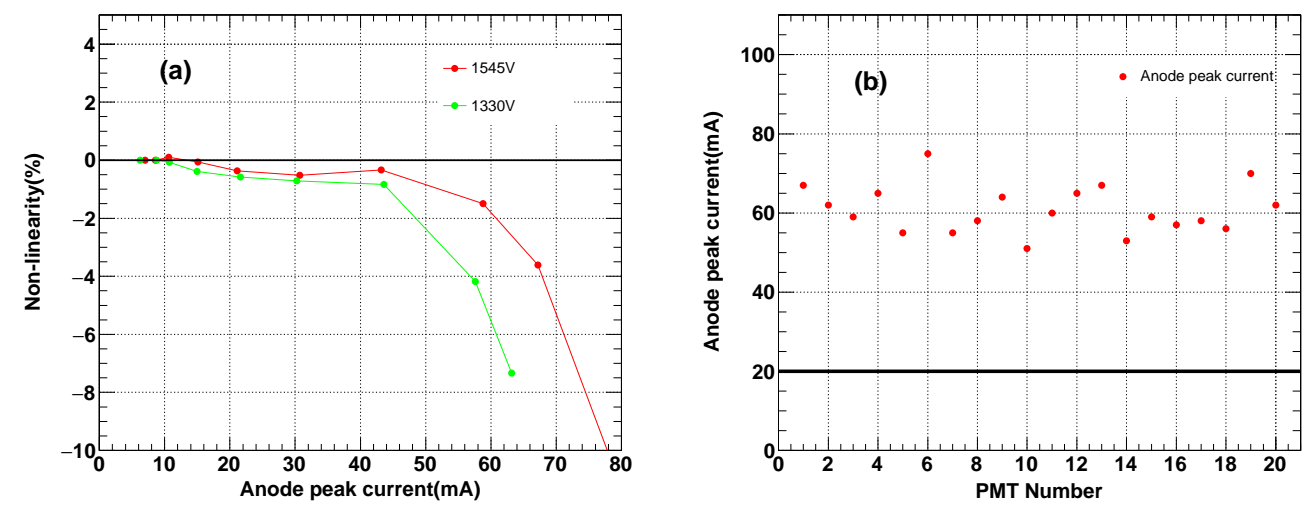

Figure 5: Figure 5(a) shows the results of the linearity measurement for a CR365-PMT at 1330V and 1545V corresponding to $3 \times 10^{6}$ gain and $10^{7}$ gain. The peak linear current is approximately $59 \mathrm{~mA}$ and $70 \mathrm{~mA}$, respectively. Figure 5(b) indicate that linearity test results of 20 CR365-PMTs are greater than $20 \mathrm{~mA}$ which also could meet our requirements.

between the two outputs of the PMT, produced by the two different intensities of pulsed light, $Q_{2} / Q_{1}$. Linearity is then calculated as follows:

$$
\eta=\frac{\left(Q_{2} / Q_{1}-Q_{02} / Q_{01}\right)}{Q_{02} / Q_{01}} \times 100 \%
$$

Figure 5(a) shows the results of the linearity measurement for a CR365-PMT at $1330 \mathrm{~V}$ and $1545 \mathrm{~V}$ corresponding to $3 \times 10^{6}$ gain and $10^{7}$ gain. In this figure, the maximum linear current is defined as the peak current where the deviation from the ideal linear current reaches $-5 \%$. The peak linear current is approximately $59 \mathrm{~mA}$ and $70 \mathrm{~mA}$ corresponding to $3 \times 10^{6}$ gain and $10^{7}$ gain, respectively. Figure 5(b) indicate that linearity test results of 20 CR365-PMTs are greater than $20 \mathrm{~mA}$ which also meet our requirements.

\subsection{Non-uniformity}

Uniformity is the variation of the output signal with respect to the photocathode position. Anode output uniformity is thought to be the product of the photocathode uniformity and the electron multiplier uniformity. In this measurement, we measure the anode output signal when LED vertically incidents on cathode's different position from $-90^{\circ} \sim+90^{\circ}$ degree along $\mathrm{X}$ axis and $\mathrm{Y}$ axis with a step of 10 degree, as shown in Figure 6. Here, non-uniformity P is defined as follows:

$$
\begin{gathered}
P=\operatorname{MAX}(P 1, P 2) \\
P 1=\left(A_{\max }-A v e\right) / A v e \\
P 2=a b s\left[\left(A_{\min }-A v e\right) / A v e\right]
\end{gathered}
$$

where the effective scanned area is considered from $-70^{\circ} \sim+70^{\circ}$ degree. Amax is the maximum anode output, Amin is the minimum anode output, Ave is the average anode output. Figure 7(a) 

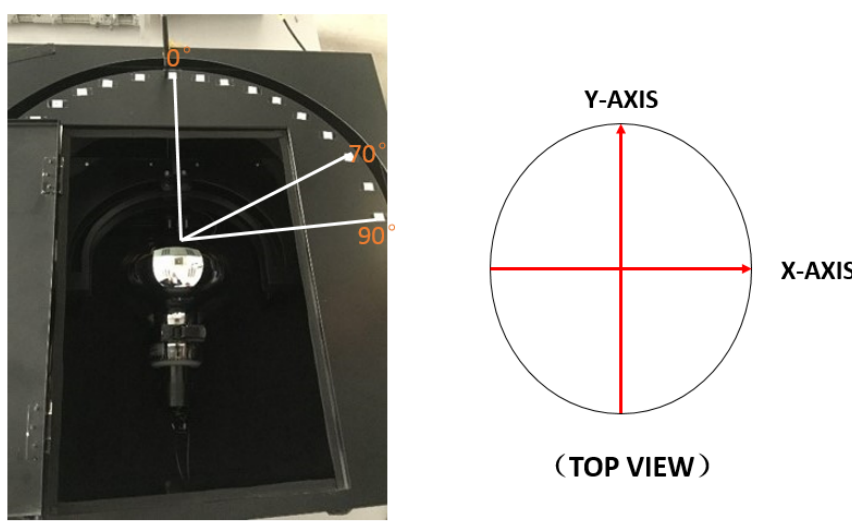

Figure 6: The anode output signal is measured when LED vertically incidents on cathode's different position from $-90^{\circ} \sim+90^{\circ}$ degree along $\mathrm{X}$ axis and $\mathrm{Y}$ axis with a step of 10 degree.
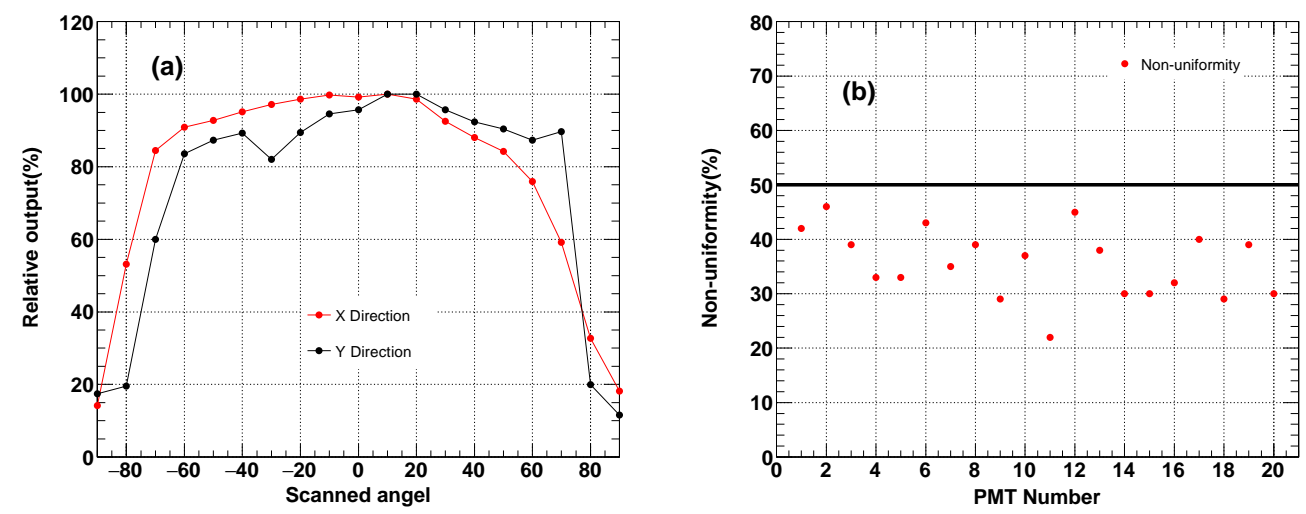

Figure 7: Figure 7(a) shows relative anode output (A/Amax) uniformity along $\mathrm{X}$ and $\mathrm{Y}$ direction. Figure 7 (b) is the non-uniformity $(\mathrm{P})$ test result of 20 CR-365 PMTs which indicate that all the 20 PMTs nonuniformity are less than $50 \%$ which is our specifiation.

shows relative anode output (A/Amax) uniformity along $\mathrm{X}$ and $\mathrm{Y}$ direction. Figure $7(\mathrm{~b})$ is the nonuniformity(P) test result of 20 CR-365 PMTs which indicate that all the 20 PMTs non-uniformity are less than $50 \%$ specified by us.

\section{Conclusions}

In this paper, we tested the characteristic features of the first batch 20 new CR365-PMT and found that they satisfy our requirements. Test results also have demonstrated that Beijing Hamamatsu company has the ability to produce this type of 8-inch PMTs stably. In the near future, we will test more new CR365-PMTs and assemble them in MD cell. 


\section{Acknowledgements}

The authors would like to express their thanks to the members of the Tibet AS $\gamma$ collaboration for the fruitful discussion. This work is supported by the National Natural Science Foundation of China (Nos. 11533007, 11673041, 11603047 and 11275212), by the Ministry of Science and Technology of China (No. 2016YFE0125500), by President's International Fellowship Initiative (PIFI), Chinese Academy of Sciences (No. 2017VMA0030), and by the Key Laboratory of Particle Astrophysics, Institute of High Energy Physics, CAS.

\section{References}

[1] M. Amenomori et al., The All-particle spectrum of primary cosmic rays in the wide energy range from $10^{14} \mathrm{eV}$ to $10^{17} \mathrm{eV}$ observed with the Tibet-III air-shower array, Astrophys.J. 678 (2008) 1165.

[2] J.R. Horandel, On the knee in the energy spectrum of cosmic rays, Astropart. Phys. 19 (2003) 193

[3] J.R. Horandel, Models of the knee in the energy spectrum of cosmic rays, Astropart. Phys. 21 (2004) 241

[4] M. Amenomori et al., Are protons still dominant at the knee of the cosmic-ray energy spectrum?, Phys. Lett. B 632 (2006) 58.

[5] M. Shibata, Y. Katayose, J. Huang and D. Chen, Chemical composition and maximum energy of galactic cosmic rays, Astrophys. J. 716 (2010) 1076.

[6] J. Huang et al., Performance of the Tibet hybrid experiment (YAC-II + Tibet-III + MD) to measure the energy spectra of the light primary cosmic rays at energies 50-10000 TeV, Astropart. Phys. 66 (2015) 18.

[7] J.-S. Liu et al., Development of Yangbajing air shower core detector for a new EAS hybrid experiment, Chinese Phys. C 39 (2015) 086004.

[8] L.M. Zhai, J. Huang, D. Chen, M. Shibata, Y. Katayose, Y. Zhang et al., Sensitivity of YAC to measure the light-component spectrum of primary cosmic rays at the "knee" energies, J. Phys. G 42 (2015) 045201.

[9] Y. Zhang, J. Huang,D. Chen et al.,Performance of new 8-inch photomultiplier tube used for the Tibet muon-detector array, JINST 11 (2016) P06016.

[10] Photomultiplier Tubes Basics and Applications. HAMAMATSU Third edition. 